\title{
Study Based on the Multi-Transactions Interface Management
}

\author{
Tu Xiang ${ }^{1,2,}$, Yang Qifeng ${ }^{1}$ and Song Ping ${ }^{1}$ \\ ${ }^{1}$ School of Economics, Wuhan University Of Technology, Wuhan, China \\ ${ }^{2}$ College of Information Engineering, Hubei University for Nationalities, Enshi, China \\ atuxiang1979@163.com
}

Keywords: Interface Management; Multi-transactions Interface; Formal Method

\begin{abstract}
Formal description method is an important approach to guarantee the accuracy of issue description. Interface management is an effective method to enhance interface efficiency among integration units. This paper proposed the conception of multi-transactions interface, and through formal modeling to the management process of multi-transactions-oriented interface, formulated the Multi-Transactions-Oriented Interface Management Model. The MTOIMM includes five aspects: Subjects Model, Transactions Model, Issues Library Model, Solutions Library Model and Multi-Transactions Interface Model. Finally, verifies the effectiveness of the MTOIMM used in the interface management of $\mathrm{O} 2 \mathrm{O}$ online payment system.
\end{abstract}

\section{Introduction}

Research on interface management originated from the studies of the capacity of scientific achievement transforming into commercial goods conducted by scholars. During the process researchers found that departmentalization of enterprises could result in reasonable labor division and clear responsibility. However, such departmentalization also leads to information barrier thus blocks commercialization process of technological achievements. Information barrier among departments is regarded as issues of interface management. In practice, failure to manage interfaces may result in additional work or in low project performance. In order to reach a common goal and mutual cooperation between project participants, need to effectively manage interface [1]. Interface described as the role and the contact between project participants. Interface issues must be exists in the interface management, Interface Issues describe the state of substance, energy and information exchange process, and Interface Issues are the sum of all the negative effects [2]. Guo et al. [3,4,5] summarized the resulting factors of Interface Issues include sticky information, target differences, cultural differences and background differences, and pointed out the important role of effective interface management for business innovation and market performance. Vandevelde \& Van Dierdonck [6] considered that different personalities, cultural differences, differences of organizational model, location factors and language problems led to the Interface Issues.

Interface Issues severely affected and restricted the development of enterprises, the survey found that when there are serious management problems in the R \& D marketing interface, $68 \%$ of the R \& D project would be a complete failure in the commercial, $21 \%$ of the project would be partially failed [7]. Chen et al. [8] regarded that Interface management failure may lead to low productivity, low product quality, and increased production costs. In order to effectively manage the Interface Issues, Guan et al. [9, 10] explained the relationship between the interface management and innovative performance. LIU et al. $[11,12]$ constructed the measure model based on the harmonious matrix in order to effectively measure Interfacial Issues. From the relationship between the starting node enterprises, the interface of the supply chain network organization is divided into market-based interface, network-based interface and enterprise-type interface [13]. Ren [14] researched enterprise cooperation and innovation from horizontal and vertical interface. Barczak [15], Wheelwright [16], \& Moenaert [17] found that in order to complete the requirements of the interface, high harmonious team is more effective than the low harmonious team.

When the effective interface management application and implementation, effective project 
management is evident [18]. The key of interface management is to accurately describe interface management process and interface management issues, and thus effectively control the interface issues in the interface management process. Therefore, we propose the concept of multi-transaction interface, and try to apply formal method to describe the general interface management process based multiple transactions interface, provide the methodology for accurately positioning and solving interface issues. The remainder of this paper is organized as follows. In section 2, proposes Multi-Transactions Interface concept, uses of formal method for the management process modeling of multi-transactions interface, establishes a Multi-Transactions-Oriented Interface Management Model (MTOIMM). After that, verifies the effectiveness of the model in Section 3. Section 4 offers some concluding remarks.

\section{Multi-Transactions-Oriented Interface Management Model}

Multi-Transactions-Oriented Interface Management Model(MTOIMM), includes Transactions Model(TM), Subjects Model (SM), Issues Library Model (ILM), Solutions Library Model (SLM) and Multi-transactions Interfaces Model (MTIM) etc., and is formally described as MTOIMM=(TM, SM, ILM, MTIM).

\subsection{Research Object.}

Each multi-transactions interface involves many subjects which refers to the summation of people, objects, organizations, processes, flows, systems or other material and energy which possess consciousness and forms. The interface this paper investigated is management interface, and the investigated issues here refers to issues of multi-transactions interface management among various subjects within one complex system. The issues between a complex system and its exterior actually can be treated as interface management issues existing among a much larger complex system and its subsystems. Hence, the research object of this paper is interface management issues among all subjects within a complex system.

\subsection{Related Concepts.}

Definition 1: One transaction is the operation process happened in the experience in order to complete a common task by the $n(n>1)$ subjects.

Definition 2: Multi-Transactions Interface is the state of interaction of materials, energies and information happened among many subjects in order to complete several relevant transactions.

\subsection{Transactions Model.}

Transactions model (TM) is the core of MTOIMM. It describes all the transactions in a complex system. There are Meta Transaction and Long Transaction in the complex system.

\subsection{Meta Transaction.}

Definition 3: Meta Transaction (MT) is the simple transaction involves only 2 subjects in the complex system. It is monodirectional and it deliver material, energy and information between sending subject and receiving subject.

MT=(ID, Name, <Sender, Receiver $>$, Description, < Material | Energy | Information>, Precursor, Successor, StartTime, EndTime, TimeConsuming, $\{$ Feature $\}$ ).

ID is the unique identification of MT; Name represents the name of meta transaction; <sender, receiver $>$ is a set of ordered pair to describe the sender and the receiver; Description is the description to the function of meta transaction; <Material | Energy| Information> describes the material, energy or information transmitted between two subjects of MT. Take the information transmitted in MT as example. Here the information refers to binary data information transmitted between computer system and is described as Information $=<$ Size, Content, Type $>$, Size is used to describe the size of information and content describes the transmitted concrete binary data; Type is the type of information, Type $=<$ structured, unstructured, semi-structured $>$, which includes structured, unstructured and semi-structured information etc.; Precursor describes the precursor of MT; 
Successor describes the successor of MT; StartTime indicates when meta transaction starts; Endtime indicates when MT ends; TimeConsuming describes the total time MT costs; \{Feature \} is the transaction characteristics set of MT, and transaction characteristics are in correspondence with the relevant interface barriers. Some explanations about the MT:

(1)MT is the minimum component of TM.

(2)In terms of the number of subjects constituting transaction, MT is the simplest type of transaction.

(3)The Sender and Receiver belong to SM.

(4) meta transaction is the simplest type of transaction.

MT is shown in figure 1.

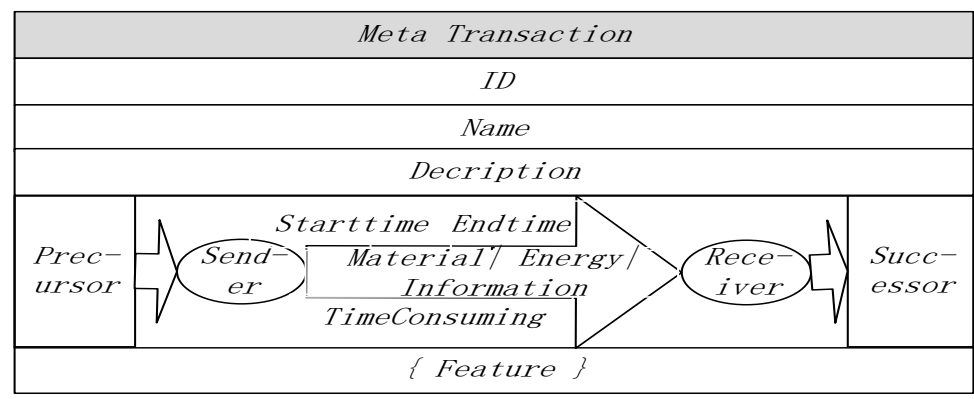

Fig.1 Meta Transaction

\subsubsection{Long Transaction.}

Definition 4: Long Transaction (LT) includes $m$ MT and $n \mathrm{LT}, m+n>1$.

LT= (ID, Participants, Method, Amount, Subtransaction, Description, $\{$ feature $\}$ )

Participants are the subjects set in the $L M$, that is $\left\{\operatorname{SubID}_{1}, \ldots, \operatorname{SubID}_{\mathrm{n}}\right\}$. Method is the Participators actions. Amount is the number of the subjects in the LT. Subtransaction $=\{M T\} \cup\{L T\}$.

Introductions to LT:

(1) The characteristics of LT are equal to the summation of the characteristics of all MTs and long transactions included in this long transaction plus the transaction characteristics owned by this long transaction itself.

(2) Each LT include n other LTs, and one LT could be included in n other LTs. It can be described in a tree-structure as follows: each LT can have $\mathrm{n}$ direct precursor LTs and $\mathrm{n}$ direct successor LTs.

(3) The inclusion relations do not exist among several LTs or between LTs and MTs included in one LT.

(4) Method in LT must be the motions of all participants involved in one transaction.

(5) One LT could not include only one MT and another LT, $m+n>1$.

\subsubsection{Transactions Model Description.}

The transaction model describes the set of all transactions in a complex system. The logical relations among all transactions of TM can be described as special tree structure, as is shown in figure 2 .

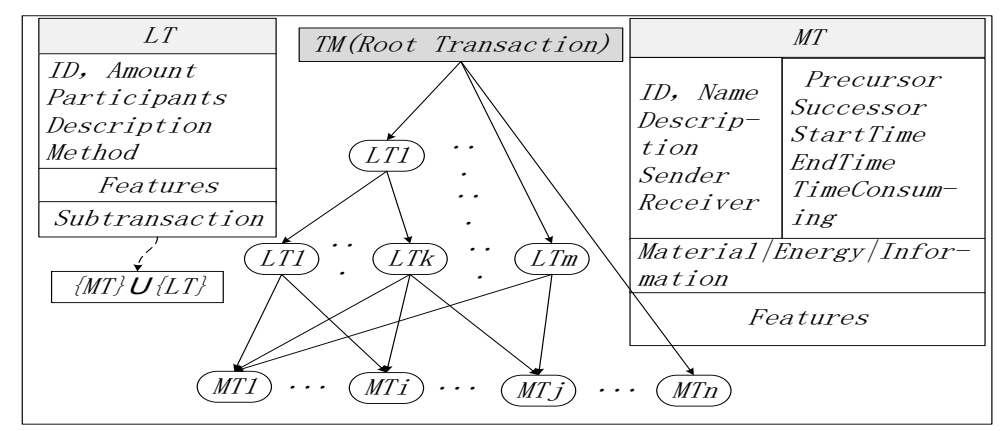

Fig. 2 Transactions Model

\subsection{Subjects Model.}


SM describes the universality and individual features of all transaction participant subjects involved in a complex system. It is a carrier of the Interface Management. The Subject described as Subject $=$ (ID, Environment, Type, Background, Features).

\subsection{Subjects Model Description.}

SM describes the set of all subjects in a complex system which possesses individual features, that is, $\mathrm{SM}=(\{$ Subject $\})$, as is shown in figure 3 .

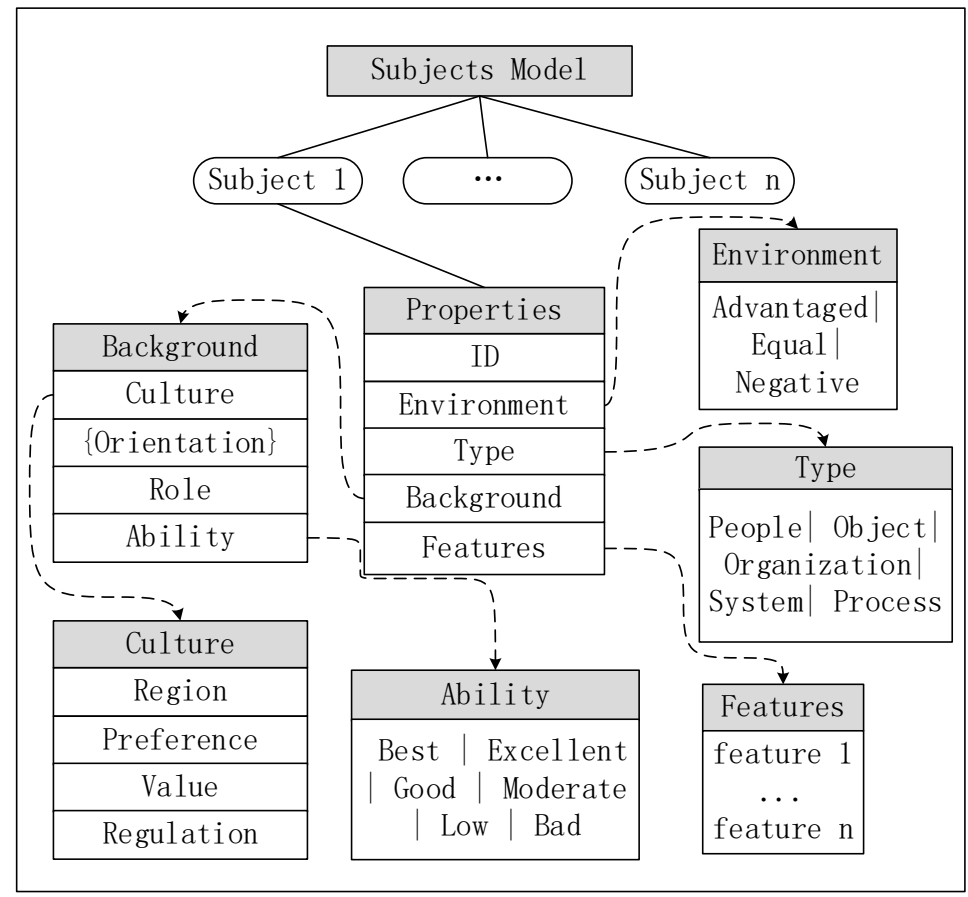

Fig. 3 Subject Model

\subsubsection{Issues Library Model.}

ILM describes the set of interface issues in a complex system, it is a strong support for effective interface management. ILM is structured by basic interface Issues. ILM is used to make new interface issues into library dynamically and dynamically upgrade new interface management plan in order to structure perfect Issues Library Model. Hence, ILM is an expertise database with large amount of knowledge and experience in interface management field. It can solve various kinds of interface issues of inner interface management field in complex systems.

\subsubsection{Interface Issues Model.}

A single issue is described as Issue = (ID, Cause, Description, Background $)$. The cause of issues is the core of Interface Issues Model (IIM). We adopted analytic hierarchy process (AHP) to make an analysis on the causes of interface issues and got several cause factors included: induced by subject's features, by requirement of transactions and by features of transmitted information, energy and material. That is to say, the cause of Interface Issues can be classified into subject cause, transaction cause and information cause, formally expressed by Cause $=<$ SI, TI, II $>$, as is shown in figure 4 .

\begin{tabular}{|c|c|c|}
\hline$I$ & $I I$ & $I I I$ \\
\hline \multirow{4}{*}{ Incentive } & $S I$ & $\begin{array}{c}G D T D M D R D \\
P D C D B D A D\end{array}$ \\
\cline { 2 - 3 } & $T I$ & $T L V R$ \\
\cline { 2 - 3 } & $I I$ & $S D A D$ \\
\hline
\end{tabular}

Fig. 4 Cause of Interface Issues

(1) SI refers to the Interface Issues induced by attribute features of participant subjects in interface, 
which included the difference of goal, type, morphology, role, preference, culture, background and ability etc. among subjects, formally expressed by $\mathrm{SI}=<\mathrm{GD}, \mathrm{TD}, \mathrm{MD}, \mathrm{RD}, \mathrm{PD}, \mathrm{CD}, \mathrm{BD}, \mathrm{AD}>$.

(2) Transaction cause refers to the Interface Issues induced by the features of transactions in interface, which includes timeliness and volume rate etc., formally expressed by $\mathrm{TI}=<\mathrm{TL}, \mathrm{VR}>$.

(3) Information cause refers to the Interface Issues induced by material, energy or information transmitted by transactions in interface, which includes standard difference and attribute difference of transmitters, formally expressed by II $=<\mathrm{SD}, \mathrm{AD}>$.

\subsubsection{Issues Library Model Description.}

ILM is described as: ILM=( Issues, addIssue(), updateIssue()), Issues= $=\{$ Issue $\}$, that is to say, ILM is the set of interface issues and includes "addIssue" method can input the new issue into library; "updateIssue" method can upgrade old issues automatically, as is shown in figure 5.

\begin{tabular}{|c|c|c|}
\hline \multicolumn{3}{|c|}{ Issues Library Model } \\
\hline \multicolumn{2}{|l|}{ Issue 1} & Issue $n$ \\
\hline \multicolumn{2}{|l|}{ id 1} & id $n$ \\
\hline \multicolumn{2}{|l|}{ GD } & AD \\
\hline \multicolumn{2}{|l|}{ Description 1} & \begin{tabular}{|l|} 
Description $n$ \\
\end{tabular} \\
\hline 15 & \multicolumn{2}{|c|}{ updateIssue () } \\
\hline \multicolumn{3}{|c|}{ Issue $1 \cdots$ Issue $n$} \\
\hline \multicolumn{3}{|l|}{71} \\
\hline \multicolumn{2}{|l|}{ New Issue } & New Issue \\
\hline \multicolumn{2}{|l|}{ Id $n+1$} & Id $n+1$ \\
\hline \multicolumn{2}{|l|}{ new incentive } & new incentive \\
\hline \multicolumn{2}{|l|}{ Description } & \\
\hline \multicolumn{2}{|l|}{ Background } & Background \\
\hline
\end{tabular}

Fig.5 Issues Library Model

\subsubsection{Solutions Library Model.}

SLM is a solutions set of the interface issues in the issues library, described as SLM=(\{Solution $\})$. Solutions library is quasi-perfect as issues library. In project management practice, SLM dynamically inputs the new solutions into library, in order to build the perfect library. Solution=(ID, InKeys, Description, Type, $\{$ IssueID $\}$ ).

A mapping relationship between the issues library and the solutions library, described as IS $=(\mathrm{I}, \mathrm{S}$, f). $\mathrm{I}=\{$ Issue $\}$; $\mathrm{S}=\{$ Solution $\} ; f=(I \rightarrow S)$. If $i \in I$ and $\left\{s_{1}, \ldots, s_{n}\right\} \in S$, then $f(i)=\left\{s_{1}, \ldots, s_{n}\right\}$, as is shown in figure 6.

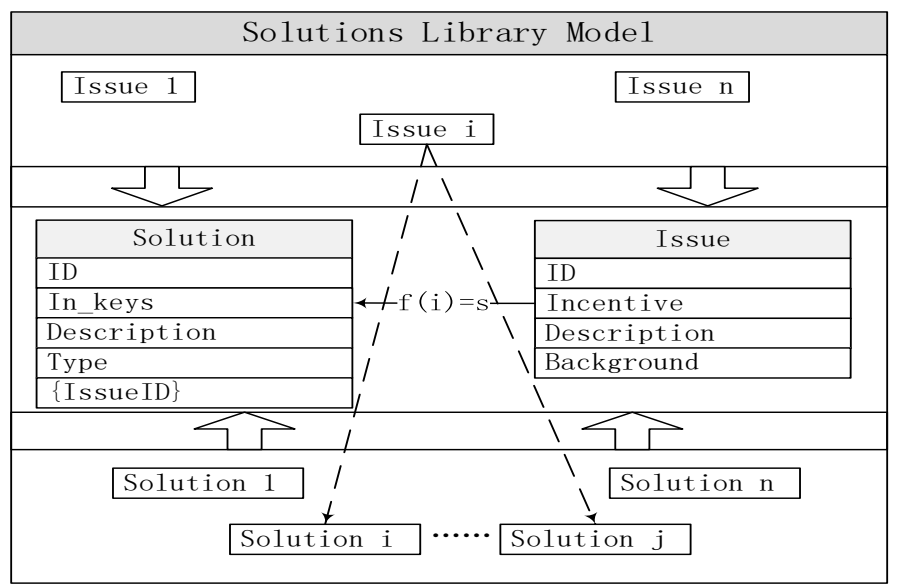

Fig.6 Solutions Library Model

\subsubsection{Multi-Transactions Interfaces Model.}

MTIM is structured on the basis of the above 4 models. 
Definition 5: Multi-Transaction Interface is:

(1) $\mathrm{T}$ is a finite set of all transactions in a complex system, $\mathrm{T}=\left\{\mathrm{t}_{1}, \ldots, \mathrm{t}_{\mathrm{n}} \mid \nexists \mathrm{t}_{\mathrm{j}} \in \mathrm{t}_{\mathrm{i}}\right.$. substransaction $\}$

(2) $\mathrm{S}$ is the set of all participant subjects in T, $S=\left\{S_{1}, \ldots, S_{n} \mid n>0\right\}$;

(3) If $t_{i} \in T \& t_{j} \in T$ and $\mathrm{Si}=\{$ ti.Participants $\} \& \mathrm{Sj}=\{\mathrm{tj}$. Participants $\}$, then $S_{i} \subseteq S \& S_{j} \subseteq S$. If $S_{i}=S_{j}$, then $\mathrm{t}_{\mathrm{i}}$ and $\mathrm{t}_{\mathrm{j}}$ belong to the same interface;

(4) $t_{i} \in\left\{t_{1}, \ldots, t_{m}\right\}, S_{i} \in\left\{S_{1}, \ldots, S_{m}\right\}, \mathrm{Si}=$ \{ti.Participants $\}$. If $S_{1}=\cdots=S_{m}$, then the $\mathrm{m}$ transactions belong to the same Long Transaction(LT), which is called Multi-Transactions Interface.

Structure Multi-Transactions Interface as follows:

Step 1: Make $T=T_{1} \cup \ldots T_{i} \ldots \cup T_{n}$, the $T i$ is the transactions set which have the same participants.

Step 2:Ti $=\{\mathrm{Ti} 1, \ldots \mathrm{Tij} \ldots, \mathrm{Tin}\}$. Tij is a LT, analyze the interface issues of Tij according to SM and ILM, and get TIi, which is one transaction interface.

Step 3: Analyze each transaction in $T_{i}$ through step 2, and get $M T I_{i}$, which is the Multi-Transactions Interfaces Model.

Step 4: Analyze each transactions set in T through step 3, and get $M T I=\left\{M T I_{1}, \ldots, M T I_{n}\right\}$.

Transaction Interface (TI) can be described as Ti = (ID, $\{$ Participant $\},\{$ Issue $\})$. Multi-Transactions Interface can be described as MTI $=<\mathrm{ID}, \mathrm{TA}, \mathrm{TP}, \mathrm{SP}, \mathrm{SA}, \mathrm{IS}>$. TA is the amount of long transactions, TP is the transaction set $\left\{T I D_{1}, \ldots, T I D_{n}\right\}$, SP is the subjects set $\left\{S I D_{1}, \ldots, S I D_{n}\right\}$, SA is the number of participants, IS is the interface issues set $\left\{I S_{1}, \ldots, I S_{n}\right\}$, and $I S=<T I D, D C, I I>$. TID is id of the transaction caused interface issue, DC is the issues description, II is an interface issue, and II $\in$ ILM.

\subsubsection{Multi-Transactions Interfaces Model Description.}

MTIM describes all multiple transactions interfaces in a complex system, MTIM=( $\{$ MTI $\})$, as is shown in figure 7.

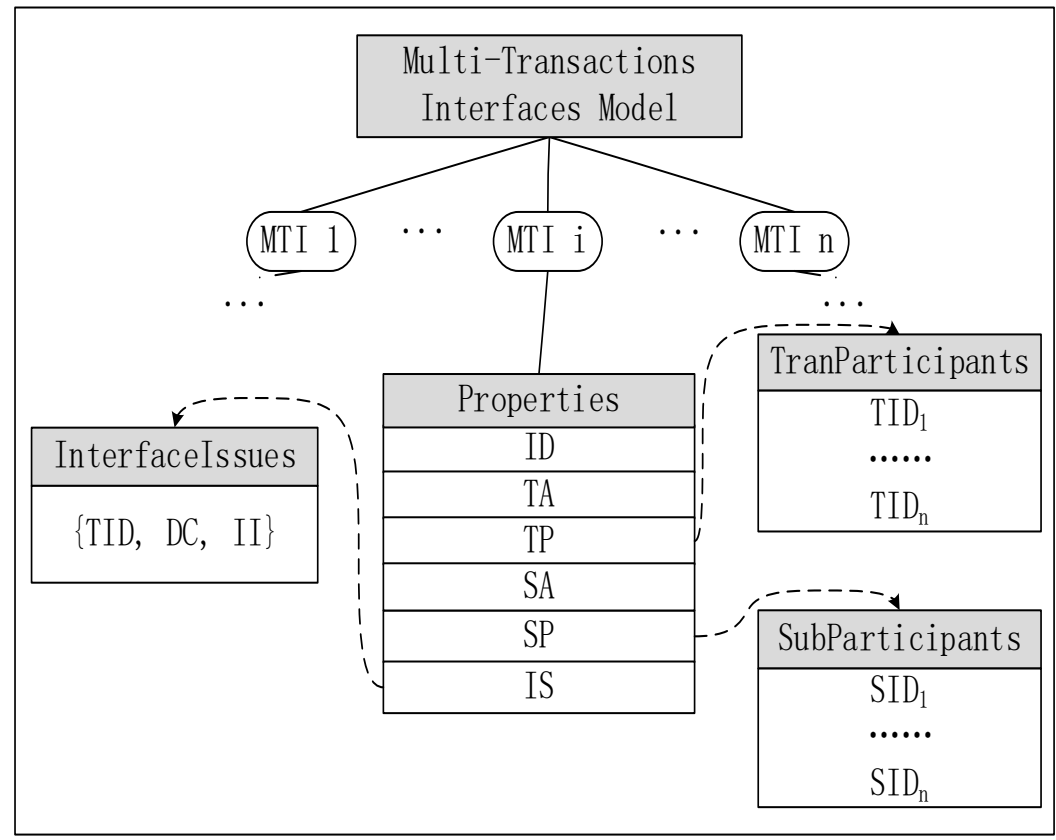

Fig. 7 Multi-Transactions Interfaces Model

\section{Model Detection}

The structure process of MTOIMM is the structure process of SM, TM, ILM and MTIM. The structure process of MTOIMM is shown in figure 8. 


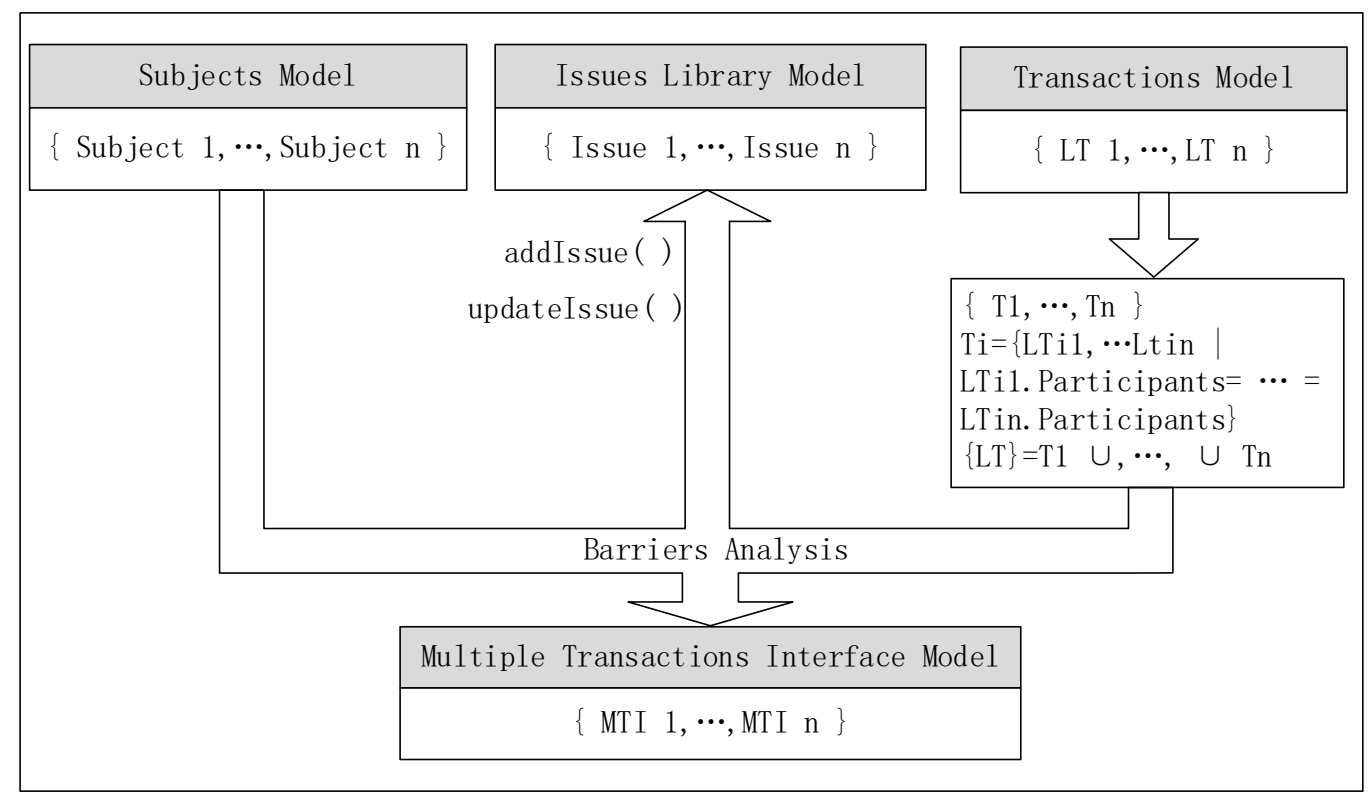

Fig.8 The Structure of MTOIMM

We define an interface management model of one $\mathrm{O} 2 \mathrm{O}$ online payment system by means of MTOIMM. O2O online payment system is a computer information management system, which includes business platform, 3-commerce platform, the third party payment platform, commercial bank and logistics management platform etc. It is a complex system, hence there must exist interface issues among inner subsystems of this system. We want to make an analysis on the probable interface issues in $\mathrm{O} 2 \mathrm{O}$ online payment system from the perspective of interface management. We take the third party platform mode as example in order to describe the interface management model of O2O online payment system. The modeling process is shown in Figure 9.

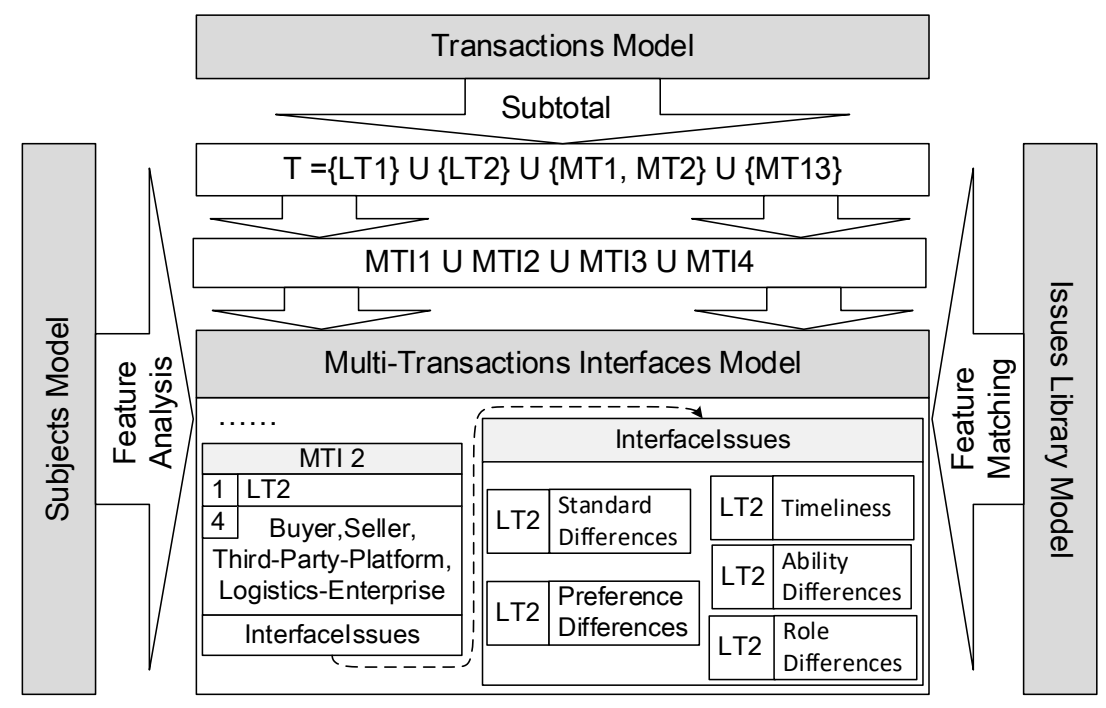

Fig.9 MTOIMM

In the figure 9, MTI2 can be described as: there is one interface participant transaction LT2 and four interface participant subjects namely Buyer, Seller, Third-Party-Platform and Logistics Enterprise. In allusion to the interface issues provided by MTI2 the multiple transactions interface, we conduct the following analysis with the combination of actual situations of online payment:

(1) If the buyer and the seller prefer different third party trading platforms, the trade may be missed due to their different preference.

(2) The interface standards of each information system haven't been unified, so the cost of development will thus increase.

(3) In the process of online payment, timely logistics service largely determines whether this trade 
could be successful.

(4) As the manufacturing enterprises, the products shipments of the upstream seller are quite large and are required to be taken to buyers in time, hence, to find a strong logistics enterprise is an important guarantee for the enterprise's sound progress.

(5) In the process of online payment, as the payment service provider, the third party payment platform possesses obvious role advantage. For example, in terms of issue of fund in transit, the payment service provider benefits more.

According to the above analysis, it is sure that MTOIMM plays an effective role in analyzing and controlling interface management issues in complex systems.

\section{Conclusion}

This paper proposed a Multi-Transactions-Oriented Interface Management Model (MTOIMM) to complex systems, abstracted the universal interface management problem in complex systems and described interface management process by formal method. The paper put forward 5 layer models of MTOIMM: Subject Model, Transactions Model, Issues Library Model, Solutions Library Model and Multi-Transactions Interface Model, described interface management model of complex systems by structuring those 5 models, which provided methodology for the analysis and control of interface issues for interface management in complex systems. Finally, the paper verified the effectiveness of MTOIMM through the modeling of $\mathrm{O} 2 \mathrm{O}$ online payment system. According to the instance analysis, it is sure that MTOIMM plays an effective role in analyzing and controlling interface management problems in a complex system.

Meanwhile, Issue Library Model should be constantly improved in the following work. In addition, transactions are always asynchronous and concurrent in the complex systems. The Petri net model can be used by subject model to describe temporal logic among transactions in the complex systems. Therefore, we can structure Petri new model of subject model to further investigate interface management issues in the complex systems.

\section{References}

[1] Guo,M.L. 2009. Study on the Financing Problem of the Middle-and-small-sized Enterprise in Perspective of Interface Management.Guide to Business, (10):71-72.

[2] LIU, B. \& SHEN, J.Q. 2012. Definition of the Concept of Interface and Interface Management. East China Economic Management, 26 (9):109-111.

[3] Guo,B. 1999. Empirical Research of Enterprise Interface Management. SCIENCE RESEARCH MANAGEMENT, 20(5): 73-79.

[4] Guo,B., Chen,J., Xu,Q.R. 1998. Interface Management: a New Thend in the Management of Enterprise Innovation. STUDIES IN SCIENCE OF SCIENCE. (1): 60-68.

[5] Guo,B., Chen,J., Xu,Q.R. 1997. Interface Management in the Process of Enterprise Innovation. Quantitative \& Technica Economics, (7): 38-41.

[6] Vandevelde, A., \& Van Dierdonck, R. 2003. Managing the design-manufacturing interface. International Journal of Operations \& Production Management, 23(11), 1326-1348.

[7] Souder, W. E., \& Chakrabarti, A. K. 1978. The R\&D/marketing interface: results from an empirical study of innovation projects. Engineering Management, IEEE Transactions on, (4), 88-93.

[8] Chen Q, Reichard G, Beliveau Y.2009. Object model framework for interface modeling and IT-oriented interface management. Journal of Construction Engineering and Management, 136(2): 187-198.

[9] Guan,J.C., Zhang,H.S., Gao,B.Y. 1997. An Empirical Study of R\&D/Marketing Interface Management. Chinese Journal of Management Science, 7(2):8-16. 
[10] Guan,J.C. \& Jin,P.A. 1995. Empirical Study on Interface Management of Firm. ECONOMIC THEORY AND BUSINESS MANAGEMENT, (6): 67-69.

[11] Liu,X.M., Xu,F.W., Zhang,Y.S. 2007. Evaluation Method Research of Effective State of Enterprise Innovation Interface, Science Research Management, (5):31—35.

[12] Liu,X.M. \& Xue,F.W. 2005. Validity Study Based on the Harmonious Interface.Technology Innovation and Management, (3):28-31.

[13] Du,Y. \& Yang,J.J. 2008. Study on Interface Management of Supply Chain Network Organizations. Soft Science, 06:63-67.

[14] Ren,R. 2010. The Integration of Cooperative Innovation and Organizational Levels Based on Interface Management Theory. Economic Management Journal, 10:180-186.

[15] Barczak, G. 1995. New product strategy, structure, process, and performance in the telecommunications industry. Journal of Product Innovation Management, 12(3), 224-234.

[16] Wheelwright, S. C., \& Clark, K. B. 1992. Organizing and leading "heavyweight” development teams. California management review, 34(3), 9-28.

[17] Moenaert, R. K., De Meyer, A., Souder, W. E., \& Deschoolmeester,D. 1995. R\&D/marketing communication during the fuzzy front-end. Engineering Management, IEEE Transactions on, 42(3): 243-258.

[18] Siao, F. C., \& Lin, Y. C. 2012. Enhancing construction interface management using multilevel interface matrix approach. Journal of Civil Engineering and Management, 18(1), 133-144. 